\title{
BMJ Open Paediatric major incident simulation and the number of discharges achieved using a major incident rapid discharge protocol in a major trauma centre: a retrospective study
}

\author{
Ruth Bird (1) , ${ }^{1}$ Daniel Braunold, ${ }^{1}$ Jack Dryburgh-Jones, ${ }^{2}$ Jordan Davis, ${ }^{2}$ \\ Sam Rogers, ${ }^{2}$ Catrin Sohrabi (i) , ${ }^{2}$ Elliot Ismail, ${ }^{2}$ Nina Mclean, ${ }^{3}$ Breda O'neill, ${ }^{1}$ \\ Naomi Edmonds, ${ }^{4}$ Rosel Tallach ${ }^{5}$
}

To cite: Bird R, Braunold D, Dryburgh-Jones J, et al. Paediatric major incident simulation and the number of discharges achieved using a major incident rapid discharge protocol in a major trauma centre: a retrospective study. BMJ Open 2020;10:e034861. doi:10.1136/ bmjopen-2019-034861

- Prepublication history and additional materials for this paper is available online. To view these files, please visit the journal online (http://dx.doi. org/10.1136/bmjopen-2019034861).

Received 10 0ctober 2019 Revised 29 July 2020

Accepted 21 September 2020

Check for updates

(c) Author(s) (or their employer(s)) 2020. Re-use permitted under CC BY-NC. No commercial re-use. See rights and permissions. Published by BMJ.

For numbered affiliations see end of article.

Correspondence to

Dr Ruth Bird;

ruth.bird3@nhs.net

\section{ABSTRACT}

Objectives Hospitals have the responsibility of creating, testing and maintaining major incident (Ml) plans. Plans emphasise readiness for acceptance of casualties, though often they neglect discharge planning and care for existing inpatients to make room for the sudden influx.

After collaboration and design of a discharge policy for a paediatric MI, we aimed to establish the number of beds made available (primary outcome) to assess potential surge and patient flow. We hypothesised that prompt patient discharge would improve overall departmental flow. Flow is vital for sick patients awaiting admission, for those requiring theatre and also to keep the emergency department clear for ongoing admissions.

Method and setting A simulated MI was declared at a London major trauma centre. Five paediatric priority 1 and 15 priority 2 and priority 3 patients were admitted. Using live bed boards, staff initiated discharge plans, and audits were conducted based on hospital bed occupancy and discharge capacity. The patients identified as dischargable were identified and followed up for 7 days.

Results Twenty-nine ward beds were created ( $42 \%$ of the total capacity). Handwritten summaries just took $13.3 \%$ of the time that electronic summaries took for the same patients by the same doctor. In-hospital transfers allowed five critically injured children into paediatric intensive care unit (PICU), and creation of a satellite PICU allowed for an additional six more if needed.

Conclusion We increased level 3 capacity threefold and created $40 \%$ extra capacity for ward patients. A formalised plan helped with speed and efficiency of safe discharge during an Ml. Carbon copy handwritten discharge letters allowed tracking and saved time. Robust follow-up procedures must be in place for any patients discharged.

\section{INTRODUCTION}

A major incident (MI) can be defined as any emergency that requires the implementation of special arrangements by one or more of the emergency services, the National Health Service or local authorities. It can involve
Strengths and limitations of this study

- Simulation allowed staff to deliberately undertake high-risk activities within a safe environment without dangerous implications.

- Learners addressed hands-on and thinking skills, putting knowledge and protocols into action, allowing real-time decision-making and effective communication to be assessed.

- Testing in real time using existing bed boards demonstrated similar results to our pilot study, suggesting validity of data.

- Simulating a large influx of admissions in a short period of time with no actual consequences of treatment mistakes or discharge decisions may produce overconfidence in discharges and overestimation of bed capacity. On top of this, major incident (MI) outof-hours discharge planning has not yet been tested.

- Posters on wards made staff and parents aware that an Ml exercise would occur. As no patients were actually discharged in the study, parental concerns and preferences were not addressed; this may be measured in future exercises.

initial treatment, rescue and transport of a large number of casualties and is likely to involve serious harm, damage, disruption or risk to human life.

MI patients are triaged on the basis of their clinical acuity, a key principle of effective MI management. Standard triage practice labels patients as priority $1(\mathrm{P} 1)$, priority 2 (P2) or priority 3 (P3). P1 patients have altered physiological parameters deemed to be life-threatening, P2 patients have non-lifethreatening vital signs but may deteriorate secondary to their injuries and P3 patients are usually ambulatory but with existing injuries.

The Pan London Major Incident guidelines state that there should be facility for 
additional critical care, and holding and treatment areas to allow for local increase in capacity and that the 'major incident plan' must allow 'mass casualty supplementation'. ${ }^{1}$ It states that, after the initial incident, the major trauma centre/tertiary unit will co-ordinate patient movement across the network in conjunction with trauma unit/district general hospital (DGH) teams to ensure patients are moved in to receive treatment and out to receive ongoing care when applicable. ${ }^{1}$

Despite these guidelines and the clear importance of planning for an MI, there are limited publications on MI planning and the testing of pathways. There are even fewer publications on discharge planning or safeguarding existing ward patients (particularly in paediatrics). This is acknowledged by Carley et al in his paper on planning for the care of children in MIs. He states, 'If casualties in major incidents are to receive the best possible care then quality planning and preparation is essential'; he also states that few hospitals plan for MIs involving children. ${ }^{2}$ Research from Challen and Walter looked at bed occupancy and predicted discharges to accommodate for an MI, but this was a retrospective study and no real-time planning was undertaken. ${ }^{3}$

MIs, which are thankfully rare events, are unfortunately occurring more regularly, and thus learning from simulated scenarios is vital. Simulation is used to substitute real experiences in a safe environment for training and planning. It replicates substantial aspects of the real world in a fully interactive manner to help promote learning and is especially useful when practising solutions to uncommon problems. Experiential learning is one of the key educational theories used to explain how simulation can support or enhance the transition from novice to expert professional practice. Kolb's experiential learning cycle explores the educational psychology underpinning learning from concrete experience. ${ }^{4}$

Johnson and Cosgrove's article on hospital's response to an MI, specifically the London $7 / 7$ bombings, looks into initial and long-term care. They highlight the significance and the challenge of the aftermath of patient care once the initial wave of patients have stopped arriving at the emergency department (ED). The Royal London Hospital stood down from the MI 5 hours after it started and reopened for trauma, but at that time theatres were operating to full capacity and the intensive care unit (ICU) had not received the patients it had already accepted from the MI. ${ }^{5}{ }^{6}$ Johnson and Cosgrove comment that 'Such actions [reopening A\&E for further trauma] have the potential to further overload pressured systems. Thus, the ongoing care of the patients admitted from the incident should form part of a major incident plan as the impact of their admission and treatment is beyond a period of a few hours'. Although widely recognised as important, most hospitals do not have a discharge protocol written in their MI policies; thus, this study aims to highlight the importance of discharge planning and to advise on a strategy.

In overhauling our paediatric MI plans in 2017-2018, we looked into key areas where we could improve admission times from accident \& emergency department (A\&E), identify key roles that help order the chaos of casualties, create targets and roles from admission wards and staff members, and not to compromise care of the patients who have already been hospitalised.

Focusing on the day-to-day pressures of EDs, an issue to overcome is 'bottlenecking'. This is where there is a build-up of patients who are unable to move onto the next stage of their treatment due to the system being overloaded. In an MI, we predicted that a rapid influx of patients on top of the regular workload would create a bottleneck for admission. Areas susceptible to bottlenecking in an MI are admissions to ED, CT scanning, theatres and paediatric ICU (PICU) and admission to wards.

The new pathway in the MI protocol is an attempt to address this issue by allowing patients to be swiftly admitted to the inpatient hospital beds, thereby freeing up more space in A\&E for the next potential wave of casualties. Rapid discharge of inpatients also allows for PICU step-downs, creating beds for incoming critically ill patients.

To allow for admissions, we created a discharge protocol, which aims to quickly and safely identify eligible patients for discharge by personnel who are not required in acute resuscitation of MI casualties. The new pathway focuses on ward doctors and nurses and places them in a bed meeting at the initiation of an MI. This allows rapid identification of which patients can be discharged or stepped down. In the daytime, the decision would be led by the consultant physician of each ward, and in the evening and weekends it would be senior nurse-led (see figure 1). Junior doctors would be split for admission/ discharge responsibilities on the wards.

Ward admissions during an MI often have a lag period, where patients are receiving treatment/interventions in $\mathrm{A} \& \mathrm{E} / \mathrm{CT} /$ theatres. We aimed to use this lag time by creating a discharge plan and completing bed clearance prior to wards being called on clinically.

We previously ran a pilot table-top exercise with live bed boards, testing the new MI policy using current inpatient demographics to assess discharge capacity in real time. Using existing bed boards and handover sheets, we were able to promptly identify patients who could be discharged, which improved the overall flow for acutely unwell patients being transferred to theatre, PICU and the ward. ${ }^{7}$

We identified discharge summaries and prescriptions as two additional key areas that slowed down the movement of patients, either from discharging them home or moving them via internal transfer. Both of these are paperwork that are usually typed and printed for each individual patient, which is time-consuming and relies on the limited resource of computers.

We developed paperwork to streamline the discharge process. We designed a 'rolling-sheet' that provides basic information about patient demographics, diagnosis, treatment and outstanding tasks for patients being 


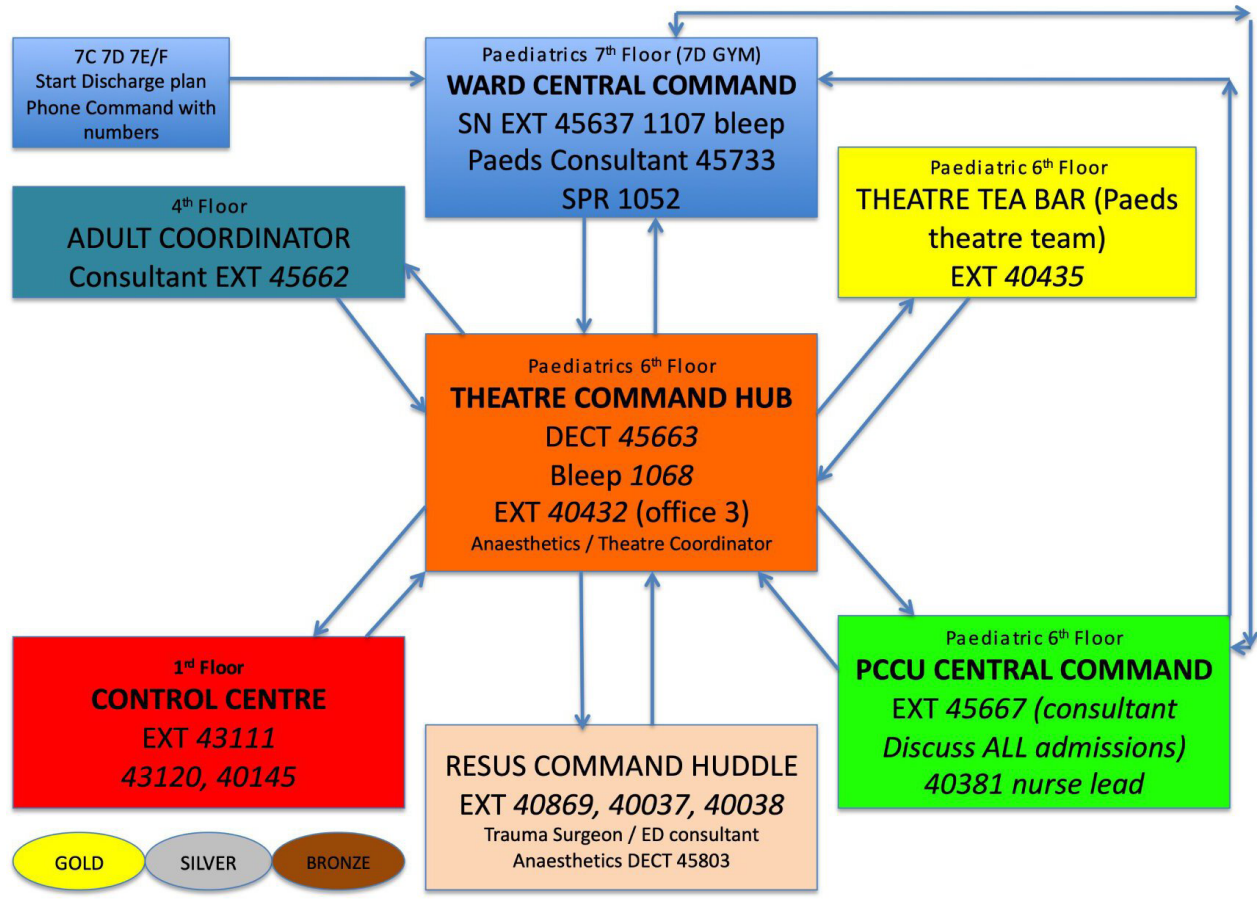

\section{MAJOR INCIDENT DECLARED}

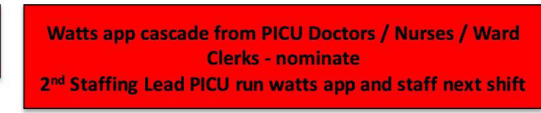

\begin{tabular}{|c|c|c|}
\hline 1. (30 minutes duration) Bed $\rightarrow$ & Location & Bed Meeting + Ward Clark \\
\hline Board meeting with senior doctor & E & Lead Nurse E \\
\hline $\begin{array}{l}\text { discharges. } \\
\text { dity }\end{array}$ & $\begin{array}{l}F \text { (to go to } E \\
\text { With board) }\end{array}$ & $\begin{array}{l}\text { Lead Nurse F } \\
\text { Consultant }\end{array}$ \\
\hline 1. PICU to identify how many & $\mathrm{C}$ & Lead Nurse \\
\hline $\begin{array}{l}\text { Step downs they have. } \\
\text { 2. Phone } 7^{\text {th }} \text { floor command with } \\
\text { Provisional Numbers as soon as }\end{array}$ & & $\begin{array}{l}\text { PASSU CONSULTANT } \\
\text { (liaise with } \\
\text { speciality consultants to start facilitating } \\
\text { discharges) }\end{array}$ \\
\hline Discharge meeting concluded. & D & $\begin{array}{l}\text { Lead Nurse } \\
\text { SPR (ward) } 1045\end{array}$ \\
\hline
\end{tabular}

3. Wards to determine how many immediate home and how many to discharge lounge awaiting pharmacy (how many staff needed for discharge lounge/to ratio of parents?) inform command how many - review which of the patients staying need RLH specifically which could repatriate to whipps etc

leads to phone pharmacy to identify estimate for TTA's from pharmacy leads to phone command with numbers - once confirmed

OUT OF HOURS DISCHARGE PLANNING NURSE LEAD

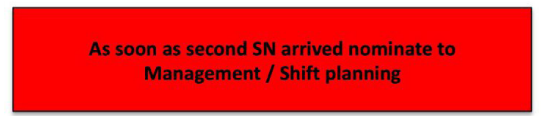

Figure 1 Major incident discharge protocol. A\&E, accident \& emergency department; CEF, Names of wards. Ward 7C 7E 7F; DW, discuss with; ED, emergency department; PICU, paediatric intensive care unit; RLH, Royal London Hospital; SPR, specilaist registra; TTA, To Take Home (written prescription).

transferred to other wards. We also produced alternative handwritten discharge paperwork with sourced printed carbon copy paper to use in place of computerised discharge paperwork. This provided the ability to transcribe and instantly triplicate the medical notes and the MI box, so a copy was provided to the patient (see online supplemental appendix for photos of equipment box).
It also greatly reduced our reliance on computers and thus enabled all paperwork to be completed quickly and efficiently.

During an MI, each ward is provided with an MI box, designed to provide a physical record of patient internal transfer and discharges. This also serves in the event of a computer failure, if the computer system cannot keep 
up with the speed of discharges, or in the event of cyber attack or IT failure.

The paperwork which is stored in the box during the MI is then transcribed after the incident to create formal electronic discharge papers and follow-ups required in the days after the exercise. Emergency prescription pads were sourced from pharmacy and placed in MI boxes, which allows patients to collect their prescriptions from their local pharmacists, allowing the bypassing of in-hospital pharmacy, which in turn frees up staff to help manage the ongoing MI.

After running the original pilot for the discharge planning in an MI event, we retested the pathway as part of a larger simulation where the hospital experienced a CBRN (Chemical Biological Radiological and Nuclear) simulation, with a large number of medical and trauma patients admitted. We re-evaluated our discharge pathway plan and tested our immediate bed capacity at ward and PICU level.

Our primary outcome measure was to understand how many beds could be created using this methodology. Our secondary outcome was the identification of the number of inappropriate discharges as a by-product of this methodology.

\section{METHOD}

An MI exercise bleep was activated at 9:45, and a message was put out on the Page One MI cascade system for permanent staff/managers. This triggered staff to take up their designated positions and job roles on the ward. As can be seen in figure 1, a collection of hubs were set up that co-ordinated admissions from A\&E, ward transfers and discharges. Each of the paediatric wards led an internal bed board meeting to evaluate how many patients could be discharged and started the process of rapid discharge. The information was passed on to a paediatric ward central command who co-ordinated movements across the paediatric hospital by taking information from PICU and resuscitation command. Information on those requiring anaesthetic aid and surgical intervention was discussed between the theatre hub and PICU hub.

Patients who were identified for discharge during the initial MI ward-based bed meetings had MI paperwork completed by doctors. Bed status and discharge numbers were audited after the exercise to assess capacity. Doctors on PICU were timed completing the discharge summaries on paper, compared with later completing the electronic version.

The MI scenario was described as a mustard gas attack that had taken place in an underground train station. During the MI, 60 patients came to A\&E, with $33 \%$ of them designated as paediatric patients. These were broken down as $5 \mathrm{P} 1 \mathrm{~s}$ and $15 \mathrm{P} 2 \mathrm{~s} / \mathrm{P} 3 \mathrm{~s}$. They received treatment and underwent any necessary imaging and tests with a time stop in ED for any patients requiring CT scanning. Patients highlighted for discharge during the exercise were not actually discharged but were followed
Breakdown of children able to be taken home immediately by parents with them on the ward after the bed board meeting.

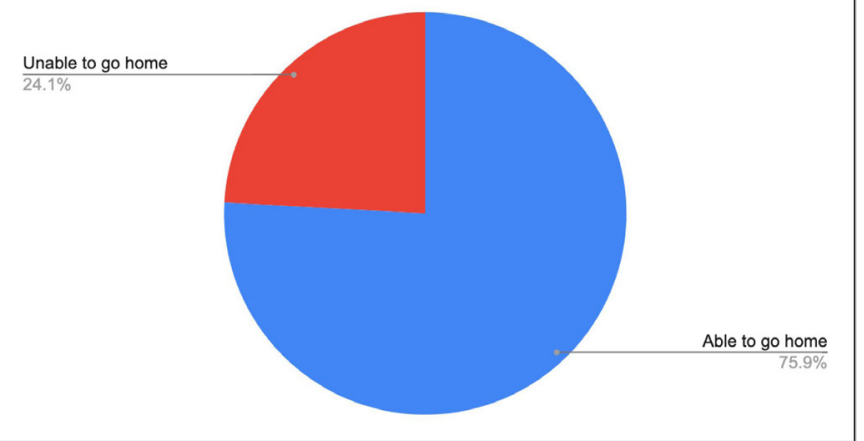

Figure 2 Breakdown of immediate versus delayed discharges.

up for 7 days to see the real date of discharge and whether there were any significant problems or readmissions during that week. No ethics approval was required for this simulation study.

The data collected included current bed board data, hospital numbers (used for follow-up, but anonymised postdata collection), age, medical/surgical conditions, outstanding discharge criteria/prescription drugs required, parental presence on the ward, simulated MI patient demographics, admission times for exercise, admission times for ward (PICU), timed call log trail, log for all PICU decision-making, staff survey results, debrief notes and timed discharge summaries.

\section{Patient and public involvement \\ No patients were involved.}

\section{RESULTS}

At the beginning of the exercise, there were a total of 68 paediatric staffed ward beds and additional five patients in the PICU.

Figure 2 shows that from the MI ward-based bed meeting, 29 patients were identified as being able to be discharged home, of whom $22(75 \%)$ had a parent with them and therefore were physically able to immediately vacate their bed. Of those 22 patients with parents, 19 required a prescription to take home, 1 required a weaning plan for inhalers and 2 required no prescriptions at all.

In PICU, two patients could be stepped down, which created three ICU beds. By doubling up nursing 2:1 until new staff could come in to help with the shift, PICU could theoretically manage up to two additional beds, making five in total.

Of the 29 patients who were selected for discharge at the bed meeting, $65 \%$ were actually discharged within 72 hours of the simulation.

The carbon copy to take home (written prescriptions) / internal transfer forms used in the exercise reduced time required for discharge from the wards dramatically. Decision-making and communication took the majority 
of the time between identification and leaving the ward. Actual completion of the paperwork took $2 \mathrm{~min}$ (which is $13 \%$ of the time taken for electronic copies transcribed later).

\section{DISCUSSION}

During the exercise, the paediatric hospital managed to identify up to 29 patients who could potentially be discharged for an incoming MI. Staff then completed the paperwork to discharge 15 patients along with two stepdown patients from PICU to the wards, allowing the admission of 20 paediatric emergency cases. This entire process took only 2 hours (the time in which the MI ran). A fully staffed discharge lounge was set up for any children able to leave but awaiting parents; this procedure is written in the discharge plans so that beds are not blocked.

We show in this exercise, and in previous pilot studies, that implementing an effective and rapid discharge system where paperwork is minimised yet tracked for completion at a later date enables a quick turnover of patients, which allows the flow of patients from A\&E to continue so that urgent care can be provided.

The discharge criteria are the following: freely clinician-led but largely included patients awaiting inpatient tests that could be performed as outpatients, patients whose elective operations or procedures were cancelled and those patients well enough to no longer require intravenous medication.

Figure 3 shows the number of days after exercise that the 29 patients identified for discharge stayed in hospital. Thirteen were discharged within 24 hours. Ten were discharged later that week. From those 10 patients, 3 were awaiting inpatient testing that could have been completed as an outpatient and could safely have been discharged home. Five of the remaining seven were on intravenous antibiotics which could have been changed to orals to allow for a rapid discharge. Of the remaining two patients who were discharged later that week, one

Follow-up from the 29 patients identified as medically fit for discharge, when they were actually discharged.

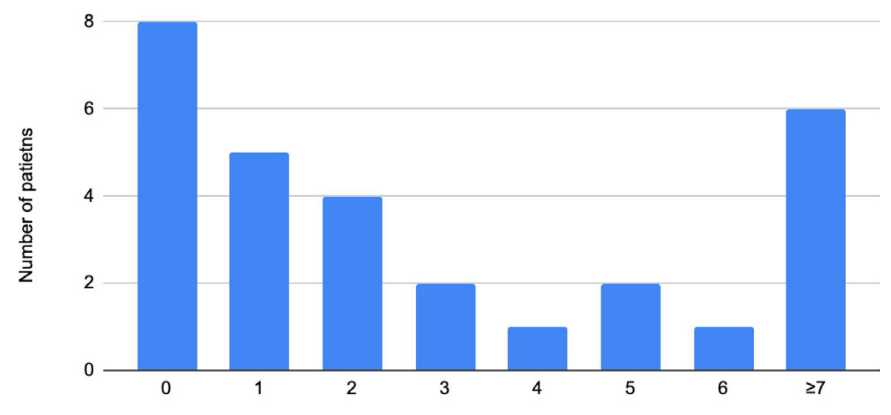

Days post major incident that potential patients that were identified were discharged

Figure 3 Follow-up of patients identified for discharge.This graph shows that in reality $58 \%$ of the children identified as able to be discharged were done so within 48 hours of the simulation. It also shows that $20 \%$ of them were still hospitalised over a week later. required diabetic education and training, which could have been community-led. The final patient was in for monitoring of a bronchiolitis flare-up but would not likely have re-presented if he or she had been discharged 48 hours earlier. There were six patients who were still in the hospital 1 week after the exercise who were identified as potential for discharge. One had cystic fibrosis that required investigations that could have been completed as an outpatient. One required ongoing blood tests to see whether his kidney injury was improving, which could have been managed by coming in for phlebotomy as an outpatient. One was not discharged due to safeguarding issue, which was only identified after the exercise. The final three patients may have been inappropriate for discharge, and if discharge had occurred, they may have bounced back into the hospital.

In light of the follow-up, it was suggested that there should be a tier system for possible dischargeable patients. Tier 1 patients would have little to no risk of re-presenting in A\&E from a failed discharge, and tier 2 patients would have a moderate risk. Using this method, the tier 1 patients would be discharged first and the tier 2 patients would only be discharged if the ongoing MI required. Despite this, in this exercise we only required 17 discharges, so the three patients who would have been inappropriate for discharge would have stayed as inpatients, adequately demonstrating that the discharge protocol for potential MI was safe and effective during this exercise.

A further cohort of patients during the study were identified as potentially transferable to the local DGHs. Although calls were not made during this MI simulation, this is a possibility in future exercises if more capacity is required.

A limitation to the methodology of simulating a mass casualty event like the one described is that the most fictitious element of the simulation is time itself. Within only 2 hours 20 paediatric admissions occurred, including 5 to PICU (including their stabilisation in A\&Eand transfer to the unit). It would require a mass of doctors, nurses, healthcare professionals and porters to do their jobs rapidly and perfectly the first time. But if one focuses on the discharge side of the equation and how it is possible to identify and start the prompt discharge process within the allocated time frame, then what is most important is that the discharge team keeps pace with the admission team.

This MI was made more challenging by simulating a CBRN incident, in this case a mustard gas attack. A large chemical injury could generate a mix of highly critically injured surgical and medical patients, with many potentially requiring the need for mechanical ventilation. It can easily be predicted that such an event would quickly deplete equipment and staff for managing such critical patients with limited PICU beds. Contingency plans should therefore be in place to help source equipment and use different areas for treating such critically ill individuals. In our scenario, we created a satellite PICU. This was where a second PICU was created for the overflow 
of paediatric patients who required ventilation. This satellite PICU can be built in paediatric recovery team and theatres using equipment from paediatric theatres, manned by theatre staff (paediatric anaesthetists, operating department practitioners and scrub nurses) and overseen by PICU consultants from the main PICU. As PICUs were able to manage the large volume (five) of patients being admitted, this service was not used on this occasion, but it could become an additional resource should the need arise.

\section{CONCLUSION}

We showed in this exercise, and in previous pilot studies, that implementing a helpful and rapid discharge system where paperwork is minimalised yet tracked for completion at a later date enabled a quick turnover of patients, thereby allowing the flow of patients from A\&E to continue so that urgent care could be provided as quickly as possible for patients in need.

The boxes (triplicate discharge forms and emergency pharmacy slips) and repeated simulation exercises streamline the discharge process, improve our ability to find capacity in an MI and prevent bed blocking and flow problems.

We believe that the simple yet practical steps that have been implemented in our trust can be quickly adapted for use in any other hospital and simulated.

\section{Author affiliations}

${ }^{1}$ Anaesthetics, Royal London Hospital, London, UK

${ }^{2}$ Barts and The London School of Medicine and Dentistry Postgraduate Studies, London, UK

${ }^{3}$ Major Incident Planning, Barts Health NHS Trust, London, UK

${ }^{4}$ PICU, Royal London Hospital, London, UK

${ }^{5}$ Royal London Hospital, London, UK

Contributors RB and DB were joint first author of the document. NM and RT led the major incident simulation for the trust. B0 and NE led on paediatric trauma, and the remaining authors were involved in the running of the simulation and data collection.

Funding The authors have not declared a specific grant for this research from any funding agency in the public, commercial or not-for-profit sectors.
Competing interests None declared.

Patient consent for publication Not required.

Provenance and peer review Not commissioned; externally peer reviewed.

Data availability statement All data relevant to the study are included in the article or uploaded as supplemental information. All data were included in the result. All related documents will be available on request to lead author.

Supplemental material This content has been supplied by the author(s). It has not been vetted by BMJ Publishing Group Limited (BMJ) and may not have been peer-reviewed. Any opinions or recommendations discussed are solely those of the author(s) and are not endorsed by BMJ. BMJ disclaims all liability and responsibility arising from any reliance placed on the content. Where the content includes any translated material, BMJ does not warrant the accuracy and reliability of the translations (including but not limited to local regulations, clinical guidelines, terminology, drug names and drug dosages), and is not responsible for any error and/or omissions arising from translation and adaptation or otherwise.

Open access This is an open access article distributed in accordance with the Creative Commons Attribution Non Commercial (CC BY-NC 4.0) license, which permits others to distribute, remix, adapt, build upon this work non-commercially, and license their derivative works on different terms, provided the original work is properly cited, appropriate credit is given, any changes made indicated, and the use is non-commercial. See: http://creativecommons.org/licenses/by-nc/4.0/.

\section{ORCID iDs}

Ruth Bird http://orcid.org/0000-0003-4396-3897

Catrin Sohrabi http://orcid.org/0000-0003-2773-5659

\section{REFERENCES}

1 Pan London mass casulatry guidence. Available: http://www.c4ts. qmul.ac.uk/downloads/network-dox/lts-mass-casualty-response.pdf

2 Carley SD, Mackway-Jones K, Donnan S. Delphi study into planning for care of children in major incidents. Arch Dis Child 1999;80:406-9.

3 Challen K, Walter D. Accelerated discharge of patients in the event of a major incident: observational study of a teaching hospital. BMC Public Health 2006;6:108.

4 Donaldson L. Safer medical practice: machines, manikins and polo mints. 150 years of the annual report of the chief medical officer. London: The Stationary Office, 2008. http://www.scirp.org/(S(i43dyn45 teexjx455q (t3d2q))/reference/ReferencesPapers.aspx?ReferencelD= 738883

5 Johnson C, Cosgrove JF. Hospital response to a major incident: initial considerations and longer term effects. BJA Education 2016;16:329-33.

6 Aylwin CJ, König TC, Brennan NW, et al. Reduction in critical mortality in urban mass casualty incidents: analysis of triage, surge, and resource use after the London bombings on July 7, 2005. Lancet 2006;368:2219-25.

7 Bird R, Lowlor J, O'Neill B, et al. Paediatric major incident planning at a major trauma centre; learning from simulation. Royal London Hospital. AMEE. 